spectrum may be due to limited exposure, although this seems unlikely.

There is some underlying suspicion that these kinds of mendelian, singlegene disorders are rare 'party tricks' thrown up by Nature that their relevance to human disease resistance is questionable. There is no question that these genes are important players in the immune response and they must underpin the basal response to infection. Do they tell us what makes a 'normal' individual resistant to the clinical effects of those pathogens that wreak most havoc on man?This is where the question "Why do many people survive infectious diseases?" comes in. Resistance to many diseases is a complex genetic phenomenon. Mycobacteria, leishmania, malaria and filaria (among others) often exert a lifelong influence on those they infect; individuals respond in a quantitative manner to infection. Twin studies show that this response is largely genetic and that the genetics is complex ${ }^{6}$. Very few of the genes underlying resistance to infection have been identified. It is likely that these genes will not be found by characterizing those rare individuals who are extremely susceptible, but by studying the complex genetics of resistant individuals. These are the genes that will yield insight into the conversion of an indifferent response to a resistant response; as such, they are coveted by scientists who seek to elucidate components of the host response. If such genes are to be found, their action must be interpreted in the context of known biology. The human gene 'knockouts' that Nature provides-as exemplified by that described by Jounguy et al.- help to contruct this biological framework. It is even conceivable that polymorphisms in such genes may increase host resistance in thier own right and as such are valuable candidates in the search for host resistance genes.

1. Jouanguy, E. et al. Nature Genet. 21, 370-378 (1999).

2. Newport, M.J. et al. N. Engl. J. Med. 335, 1941-1949 (1996).

3. Jouanguy, E. et al. N. Engl. J. Med. 335, 1956-1961 (1996)

4. Huang, S. et al. Science 259, 1742-1745 (1993)

5. Young, H.A. \& Hardy, K.J. J. Leukocyte Biol. $\mathbf{5 8}$ 373-381 (1995).

6. McLeod, R., Buschman, E., Arbuckle, L.D. \& Skamene, E. Current Op. Immunol. 7, 539-552 (1995).

\title{
A vital role for vitamin A
}

\section{Gregor Eichele}

M ax Planck Institute of Experimental Endocrinology, Feodor Lynen Strasse 7, 30625 H annover, Germany

Back in the early 'eighties when the molecular basis of embryonic development was largely unchartered territory, the discovery that retinoic acid can change patterns of $\operatorname{limb}^{1}$ and nervous-system ${ }^{2}$ development was greeted with astonishment. The molecule achieved celebrity status once its endogenous presence was detected in the embryonic tissues that it affects ${ }^{3}$ and its status as a ligand to a family of nuclear receptors ${ }^{4}$ (that bind DNA and regulate the expression of target genes) was established. The early 'nineties brought to the fore numerous new peptide signalling molecules at breath-taking speed. The hedgehog, W nt, FGF and TGF signalling molecules competed with cancer genes for the attention of those perusing the literature.

Genetic dissection of the biology of the retinoid receptor molecules was attempted by generating 'knock-out' mice ${ }^{5}$, but this was confounded by the fact that mammalian genomes contain six genes encoding two classes of receptor. Mutations in a single receptor gene resulted in-at most- mild aberrations in developmental phenotype, although compound mutants demonstrated more marked developmental defects. After a great deal of heroic work, one was left with the impression that retinoids play a role in animal development, but as an 'extra', on a stage ruled by clans of hedgehogs, FGFs, TGFs and
W nts. A study presented on page 444 of this issue by Karen Niederreither and colleagues corrects this notion ${ }^{6}$.

Animal cells cannot manufacture, de novo, retinoids; they ingest them in the form of vitamin A, also known as retinol, and then convert retinol, via retinal, to retinoic acid. These reactions are catalyzed by specific enzymes, some of which have been isolated and cloned. Raldh2, which converts retinal to retinoic acid, is one such example ${ }^{7,8}$. Its expression pattern in the

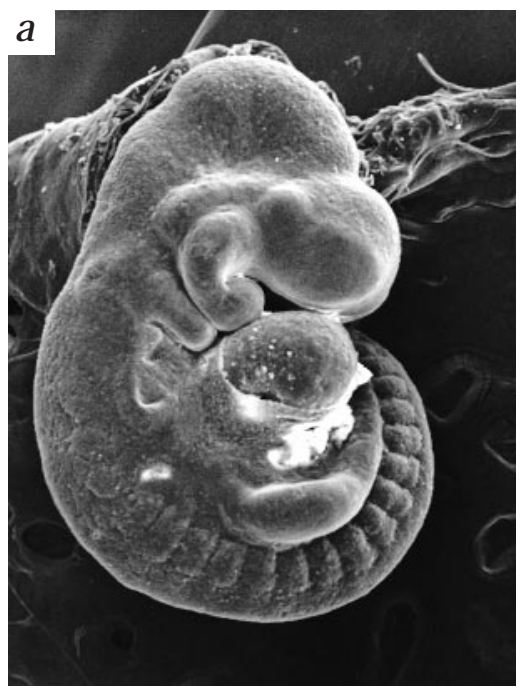

Fig. 1 Scanning electron micrographs of normal (a) and Raldh2-/- (b) mice, aged 9.5 days. Images kindly provided by Pierre Chambon and colleagues.

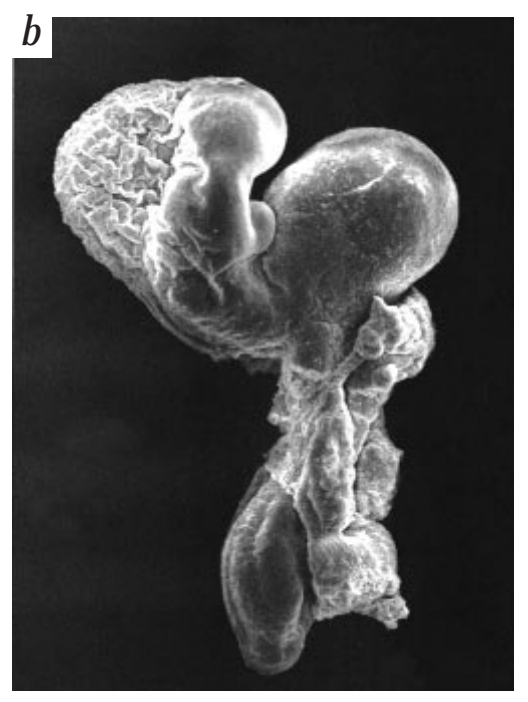

mouse embryo suggests that it is required for the local production of retinoic acid 9 . One might predict that inhibiting the localized production of retinoic acid would arrest-at least in some parts of the embryo - the retinoid signalling pathway, and thus unmask the role of retinoids in development. The study by Niederreither et al. goes some way to confirming this pre diction, in that it demonstrates that mice deficient in Raldh2 have major developmental defects and die mid gestation nature genetics • volume $21 \bullet$ april 1999 


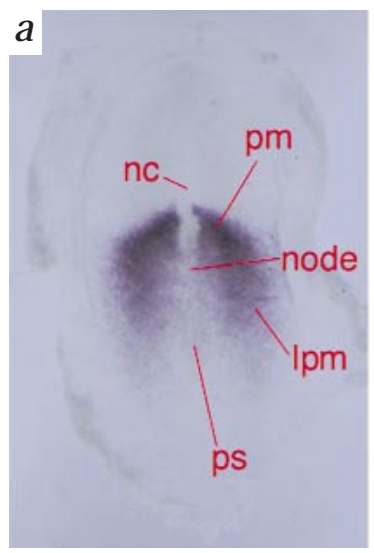

b

(Fig. 1). Posterior mesodermal structures are severely affected and those that do develop are diminutive. Limb buds never appear, and Fgf8 and Fgf10, markers that delineate the limb region prior to limb bud formation, are not expressed in the flank. Mutant embryos also have severe heart defects, and lack certain facial structures. Niederreither et al. provide bona fide evidence that Raldh2 ${ }^{-l-}$ embryos are devoid of retinoic acid-they crossed a retinoidresponsive LaCZ reporter transgene into the null mice, and could not detect $\beta$ galactosidase. This suggests that Raldh- 2 is the primary enzyme that catalyzes the conversion of retinal to retinoic acid. It could be argued that signalling molecules other than retinoic acid require Raldh-2 for proper processing; provision of in utero retinoic acid results in partial rescue of Raldh2-I- embryos but some of the abnormalities persist-albeit with less severity.

Is there a unifying explanation of the plethora of defects in trunk mesodermal structures (somites, limbs and heart) observed in Raldh2 mutant embryos? Ear-

Fig. 2 Raldh2 expression (purple) shown in a 1-day-old chick embryo (a) and superimposed onto an equivalent stage fate map of mouse (ref. 17; b). Prospective paraxial mesoderm (pm) and lateral plate mesoderm (lpm) strongly express Raldh2 and give rise to structures such as somites, head mesenchyme and limbs that are severely affected or absent in Raldh2 mutant mice. Notochord (nc), Hensen's node, and the primitive streak (ps) are indicated. The anterior part of the embryo is positioned at the top of each figure. Image provided in panel (a) kindly provided by Eric Swindell.

lier work suggests that posterior tissue synthesizes retinoic acid $^{10}$. A schematic fate-map (Fig. 2) superimposed on the Raldh2 expression pattern illustrates the fact that affected mesodermal structures arise from tissues expressing Raldh2. For example, somites derived from mesodermal cells located adjacent to the primitivestreak and limb buds develop from more laterallypositioned mesoderm. The data obtained by Niederreither et al. clearly show that retinoic-acid signalling is required for the proper development of early embryonic mesoderm. Exactly how it mediates development remains to be determined, but establishing the identity of the retinoid responsegenes in thetrunk mesoderm will obviously shed light on the matter. It is likely that Hox genes are targets-it's common knowledge that retinoic acid regulates Hoxal expression; consistent with this is the dramatic reduction of Hoxal expression in Raldh2 mutant embryos.

The early death of Raldh2-/- embryos prevents deduction of developmental processes mediated by retinoic acid that occur later in life. For example, spinal motor neurons use a retinoid-mediated signal to specify the identity of a derivative subset of motor neurons ${ }^{11}$. Studies using a retinoid receptor antagonist implicate retinoic acid in specifying the zone of polarizing activity, a signalling center in thelimb bud ${ }^{12}$. Retinoid signalling has also been implicated in mediating the development and function of the dopaminergic system of the mammalian brain ${ }^{13,14}$ and in long-term potentiation and depression ${ }^{15}$; there are hints that aberrant retinoid signalling may contribute to schizophrenia ${ }^{16}$. While we have been aware of retinoids for the better part of a century, our knowledge of their action is itself embryonic. As testified by the current study, application of molecular tools continues to advance our understanding.

1. Tickle, C. et al. Nature 296, 564-546 (1982).

2. Durston, A.J. et al. Nature 340, 140-144 (1989)

3. Thaller, C. et al. Nature 327, 625-628 (1987).

4. Mangelsdorf, D.J. et al. Cell 83, 835-839 (1995).

. Kastner P. et al.Cell 83, 859-869 (1995).

6. Niederreither K. et al. Nature Genet. 21, 444-448 (1999).

7. Zhao, D. et al. Eur. J. Biochem. 240, 15-22 (1996)

8. Whang et al. J.Biol. Chem. 271, 16288-16293 (1996).

9. Niederreither K. et al. Mech Dev. 62, 67-78 (1997).

10. Hogan, B.L. et al. Nature 359, 237-241 (1992).

11. Sockanathan S. et al. Cell 84, 503-514 (1998).

12. Helms, J.A. et al. Development 122, 1385-1394 (1996).

13. Zetterstrom R.H. et al. Science 276, 248-250 (1997)

14. Krezel W. et al. Science 279, 863-867 (1998)

15. Chiang M.Y. et al. Neuron 21, 1353-1361 (1998).

16. Goodman, A.B. Proc. Natl Acad. Sci. 95, 7240-7244 (1998).

17. Smith, J.C. et al. Devel. Dynamics 201, 279-289 (1994).

\title{
Making sense out of sound
}

\author{
Andrew J. Griffith \& Thomas B. Friedman \\ Laboratory of M olecular Genetics, National Institute on Deafness and Other Communication Disorders, National Institutes of H ealth, 5 Research Court, \\ Rockville, M aryland 20850, USA. e-mail: friedman@nidcd.nih.gov
}

Sound is a well-understood, physical phenomenon. How we perceive sound and make sense of complex auditory stimuli is a different matter altogether. Genetic approaches, however, have provided a powerful strategy to dissect auditory function and to some extent, overcome the challenge posed by the inaccessibility and scant quantities of cochlear neurosensory tissue lying deep within the temporal bone. Although remarkable progress has been made in identifying genes causing hearing loss, correlating the functions of those genes with specific auditory processes or pathways is not always straightforward. On page 363 of this issue, Shin'ichiro Yasunaga and colleagues report the latest 'deafness' gene' ${ }^{1}$. While its function has yet to be determined, its sequence and expression in the inner hair cells of the cochlea provide some intriguing clues.

The cochlear neuroepithelium includes inner hair cells (Fig. 1). These transduce sound into electrical impulses that are transmitted to the central nervous system. While transducing sound, the cochlea faithfully preserves the frequency information of the stimulus. It accomplishes this, in part, by a tonotopic 